\title{
EXISTENCE AND INCIDENCE OF OSTEOID IN OSTEOARTHRITIC FEMORAL HEADS
}

A Preliminary Report

\author{
H. C. Batra, Glasgow, Scotland, and J. Charnley, Wrightington, Lancashire, England
}

From the Centre for Hip Surgery, Wrightington

In the course of routine histological examination of femoral heads excised in cases of osteoarthritis of the hip treated by total replacement arthroplasty, osteoid was often found in the cancellous bone. In further studies to determine the incidence and orientation of osteoid in such specimens forty femoral heads were examined. Sections were prepared by the method of Ball (1957) using low viscosity nitrocellulose. All sections were made in duplicate and stained with von Kossa and haematoxylin and eosin stains.

\section{OBSERVATIONS}

All the specimens available to us were from patients in the seventh and eighth decades with advanced osteoarthritis of the hip needing radical surgery. Invariably, they showed gross macroscopic changes such as cartilage destruction, eburnation and vertical collapse. Such changes were most pronounced over the pressure-bearing segments of the heads. Diffuse velvety change with a pinkish hue or circumscribed erosive appearance of the cartilage was confined mostly to surfaces that were not pressure-bearing.

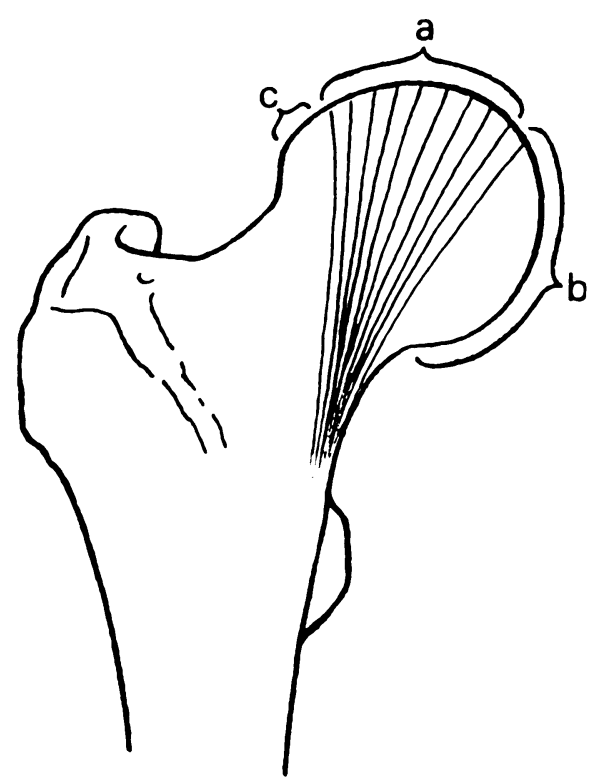

Fig. 1

Diagram showing division of the femoral head into "pressure bearing" (a) and "nonpressure " bearing $(b)$ and $(c)$ segments. (After Harrison, Schajowicz and Trueta, Journal of Bone and Joint Surgery, 35-B, 598.)

Osteoid was present in the cancellous part of the bone in twenty-seven of the forty specimens. No definite pattern was seen in different specimens or even in the same specimen, either in the amount or extent of osteoid. We did not attempt to measure quantitatively the amount of osteoid in any of these sections. In some cases it was widespread whereas in others it was limited to a few trabeculae. The size of the seams was also variable. However, a thorough search was made for it in every specimen, and in many cases in more than one section from the same specimen. In each case, osteoid was confined within the limits of the "pressure segment" and no osteoid was seen in the "non-pressure segment " of the femoral head.

The terms "pressure segment" and "nonpressure segment" were first used by Harrison, Schajowicz and Trueta (1953) as subdivisions of the femoral head according to its trabecular architecture. A relatively dense group of trabeculae (Fig. 1) runs down a broad surface of the femoral head into the neck, forming a cone with its base at the surface of the femoral head. These trabeculae are concerned with the direct transmission of body weight and have been called the "pressure system," and the segment of the femoral head that they occupy or traverse is called the "pressure segment." The rest of the femoral head has much fewer pressure-bearing trabeculae and is called, therefore, the "non-pressure segment." These terms are used in this paper and the two distinct zones were oriented when our sections were prepared. 
The presence of osteoid in the sections must be seen against a background of marked bone atrophy, a finding common to both the non-pressure and pressure segments. Atrophy was more marked in the latter, with a resulting decrease in the number and thickness of bony

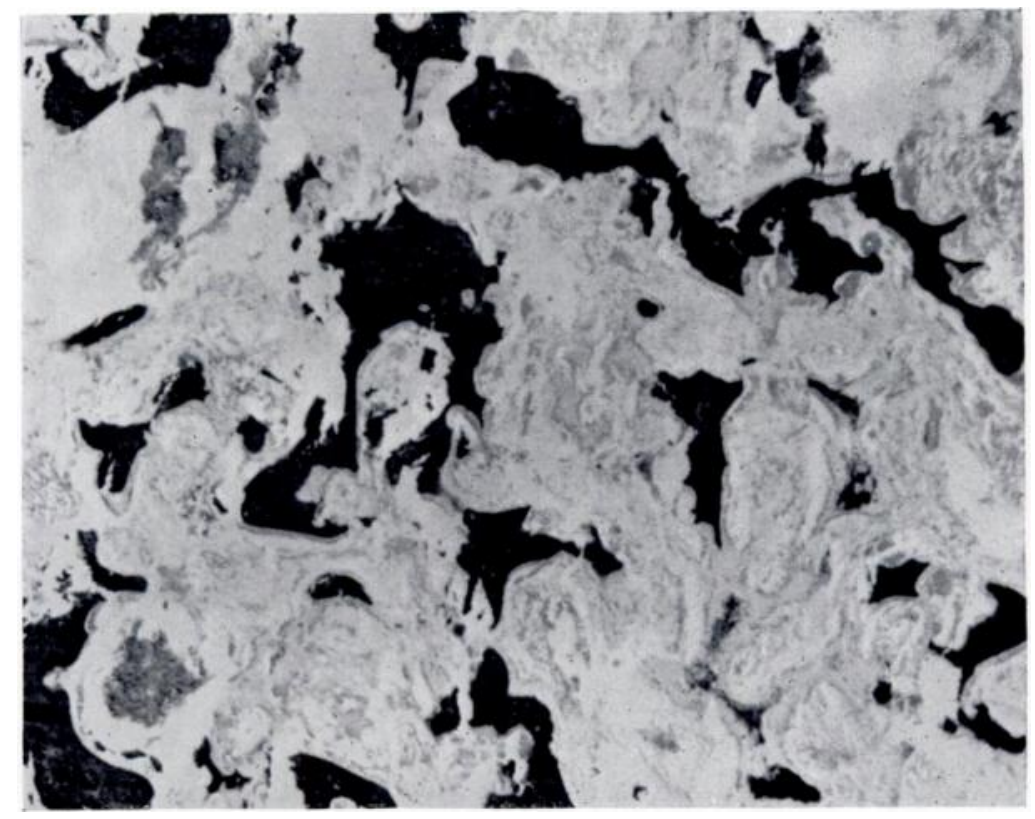

Fig. 2

Section showing the degree of cancellous bone atrophy with fibrous dysplasia and irregular osteoid formation. (Undecalcified bone; von Kossa stain; low power.)

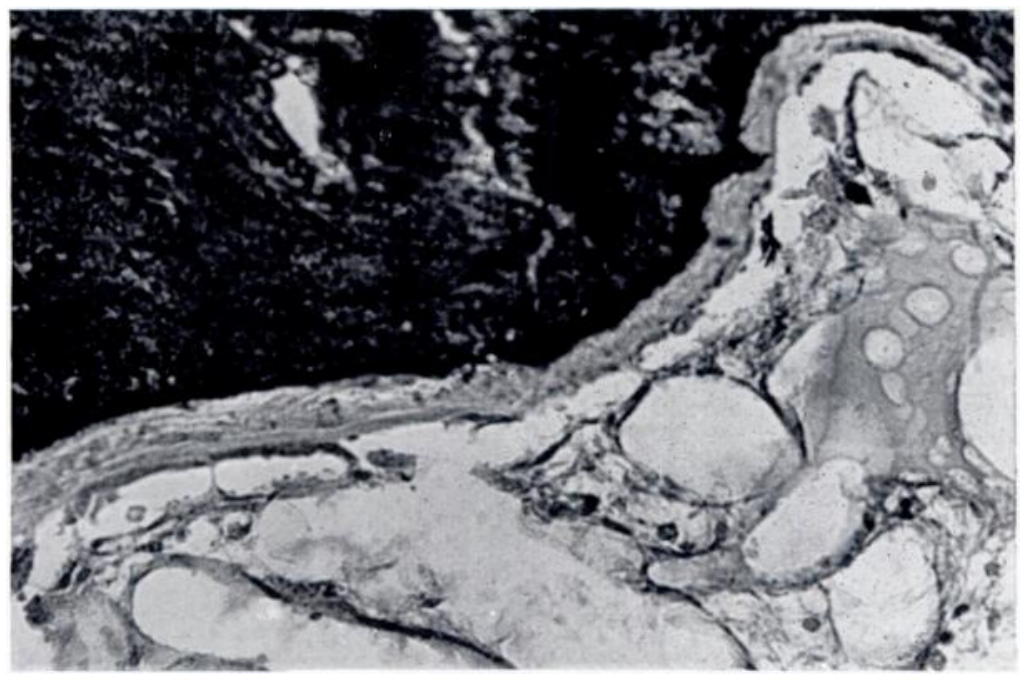

Fig. 3

Section of subchondral bone in an osteoarthritic femoral head showing a continuous osteoid seam. (Undecalcified bone; von Kossa stain, $\times 75$.)

trabeculae and with large intertrabecular spaces only partly filled with altered marrow and fibrous tissue. The normal meshed architecture of interconnected trabeculae was lost and huge gaps seemed to separate them (Fig. 2). Some of these trabeculae showed attenuation to the point of fracture, while a few lay free, having undergone aseptic necrosis with 
characteristic loss of osteocytes and relative sclerosis. Dead bone near the subchondral zone remained in situ. A marked fibrous tissue reaction surrounded it and sometimes osteoclastic resorption was seen.

In all twenty-seven cases, osteoid seams were more numerous and larger in the immediate subchondral part of the bone. In fifteen they continued deep into the cancellous bone of the

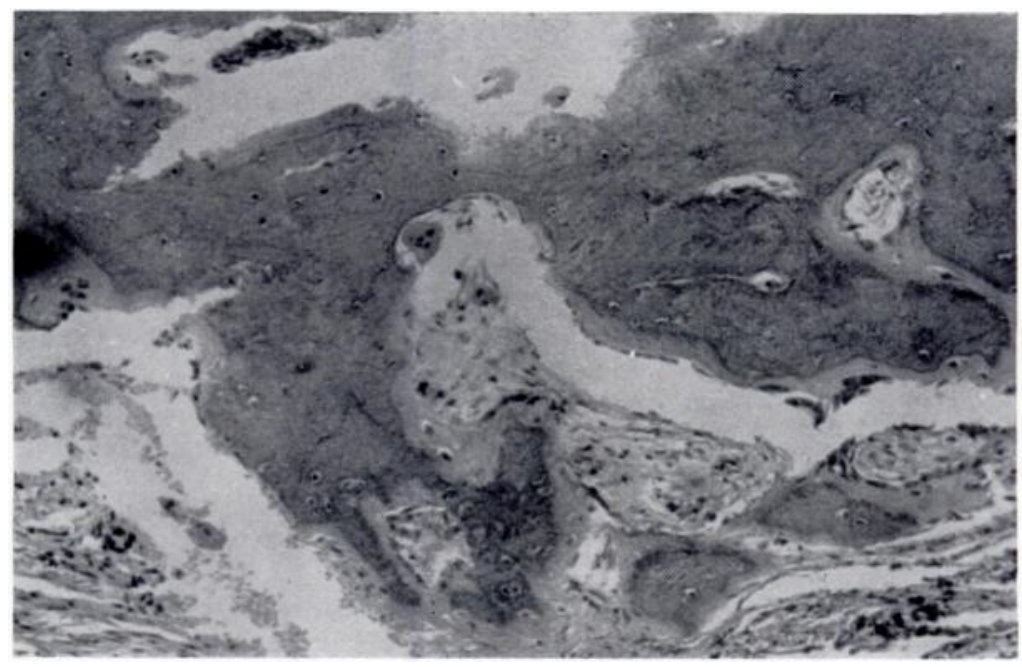

FIG. 4

Section showing osteoid and osteoblastic activity. (Undecalcified bone; haematoxylin and eosin, 75 .)

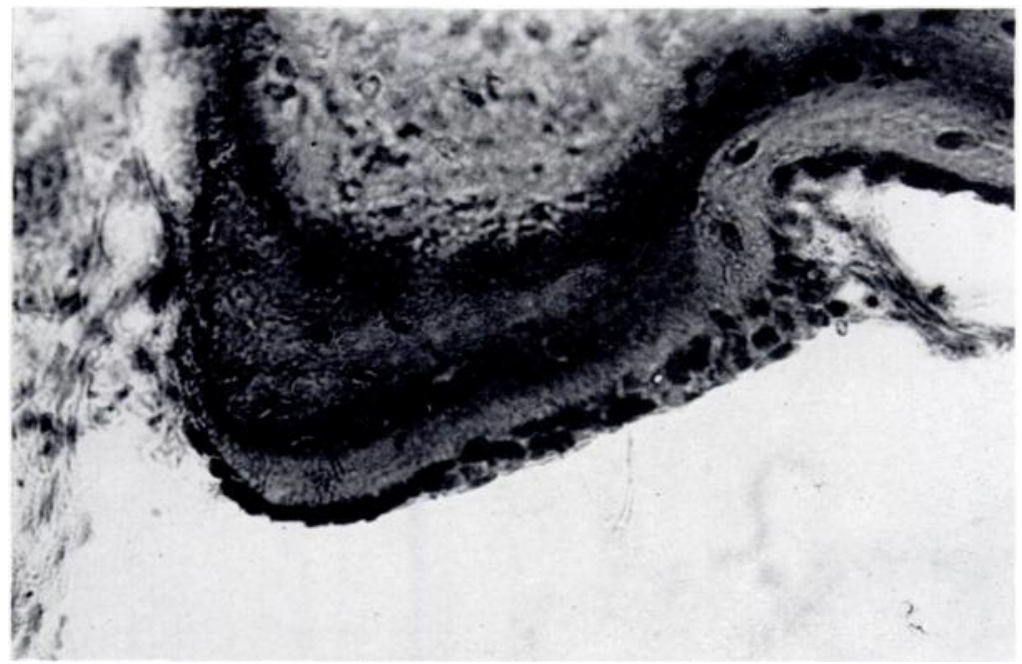

Fig. 5

Magnified photomicrograph of a bony trabeculum showing osteoid and multilayered osteoblastic hypertrophy. (Undecalcified bone; haematoxylin and eosin, $\times 145$.

rest of the pressure segment. In the other twelve they became scanty and thinner as they receded from the subchondral zone. Localised areas with large amounts of osteoid and others without it were occasionally encountered (Figs. 3 to 5).

The osteoid seen in our specimens was not like that found in a typical case of osteomalacia. In osteomalacia it is almost always distributed uniformly in the cancellous bone and is not associated with prominent layers of large active osteoblasts lying just outside the osteoid 
seams which they had laid down. In normal bone not only is osteoid not seen (or if it is, it is in extremely small amounts, ordinarily undetectable), but the osteoblastic activity is at a very low ebb and a single row of osteoblasts, widely separated from each other, lies along the trabecular margin. These are the so-called " resting osteoblasts."

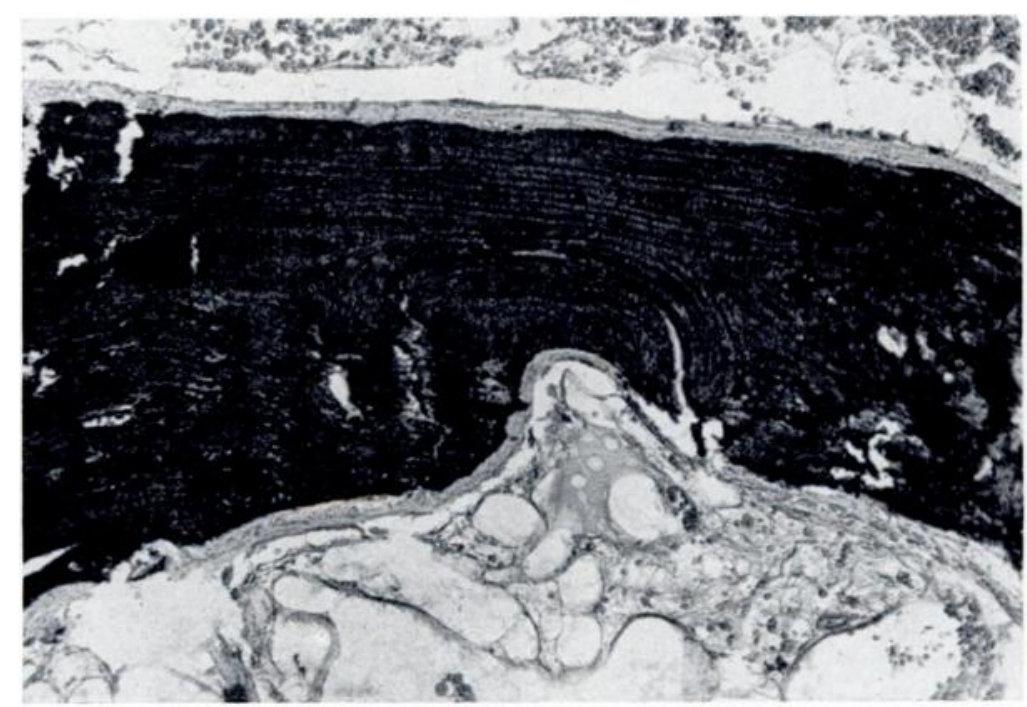

Fig. 6

Intense fibrous tissue degenerative appearance in a bone section from an osteoarthritic femoral head. The production of osteoid is more marked closer to this fibrous tissue. (Undecalcified bone: von Kossa stain, $\times 75$.)

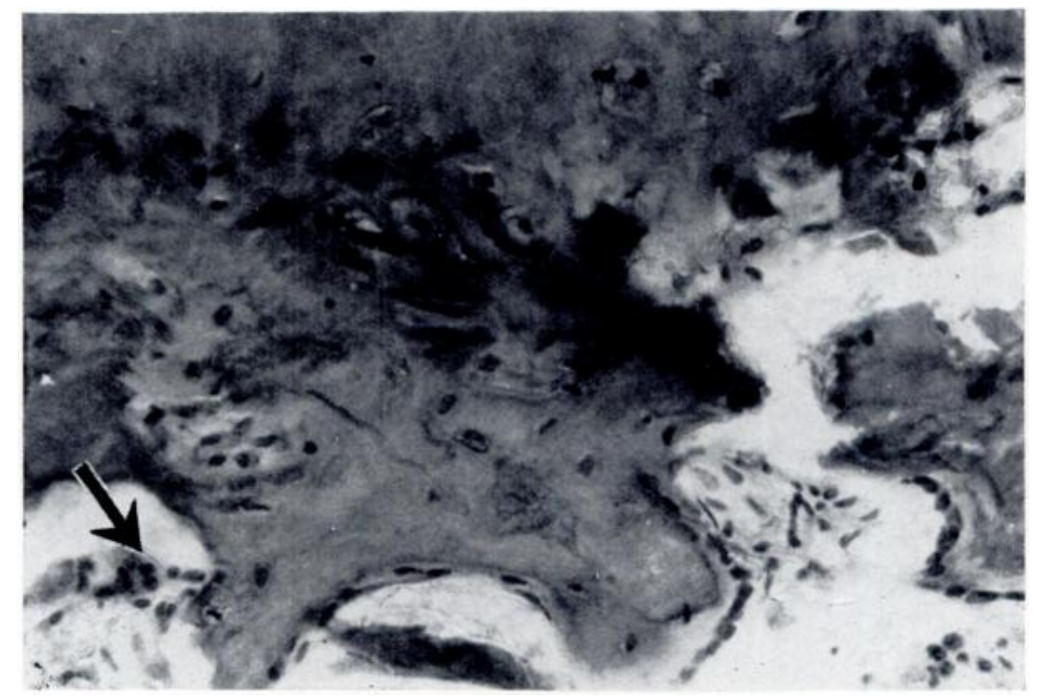

FIG. 7

An osteoclast (see arrow) lying just outside the bony trabeculum at a site with no osteoid formation. Osteoid is seen in other parts of the section. (Undecalcified bone; haematoxylin and eosin, $\times 145$.)

A greater degree of osteoblastic reaction was found in trabeculae lying in close proximity to the excessive fibro-vascular tissue filling the enlarged intertrabecular spaces. These so-called "cysts" give the impression of an attempt to overcome the barriers to bony repair put up by the fibrous tissue. The process is, perhaps, a reaction to degeneration (Fig. 6). 
Osteoclasts were seen less frequently and only in association with dead bone trabeculae (Fig. 7). This is not surprising because it has been observed before that surfaces covered with osteoid are protected by it from erosion by osteoclasts, although sometimes the osteoid sheath may be breached by active osteoclasts via a marrow tunnel carrying fibro-vascular tissue from the marrow to the mineralised core (Garner and Ball 1966).

\section{COMMENT}

It is generally accepted that osteoid is young bone prior to calcification: that is, it is bone matrix already recognisably differentiated but not mineralised (Collins 1966). It is a characteristic finding in osteomalacia and its presence is an index of the lag in calcification in this condition (McLean and Bloom 1940).

Deposition of mineral normally follows so quickly upon the formation of bone matrix that even the electron microscope can distinguish only an extremely thin zone of osteoid around calcified bone. Delay between elaboration of matrix and its calcification is due either to exceptionally rapid osteoid production, as in parts of an osteogenic sarcoma, or to the relative unavailability of calcium and other agents necessary for calcification, as in rickets and osteomalacia.

The occurrence of osteoid in osteoarthritis has, we believe, never been reported before although attempts at partial healing and repair of bone inside femoral heads have been described (Harrison et al. 1953). Its presence and high incidence in our sections may be consistent with the theory of intrinsic repair in the cancellous bone of the pressure segment where maximal degenerative changes are seen. Judging from the degree of hypertrophy and increase in the number of osteoblasts, it appears that osteoid is a manifestation of the reparative processes within the areas of greatest stress, and that it is the result of osteogenesis taking place in response to degenerative change. Why this new bone is not immediately calcified is obscure. In other words, why is so much osteoid seen when in other conditions involving bone repair mineralisation of newly laid bone matrix proceeds parallel with or follows immediately upon the genesis of collagen matrix, except of course in metabolic disorders such as osteomalacia and rickets the reasons for which are well established?

The explanation must lie between two possibilities or in a combination of both. Either a low blood mineral level obtains locally in the arthritic femoral head or a very rapid and exuberant osteoid formation occurs and this exceeds the mineralising capacity of the normal blood levels of calcium.

In a consideration of the first alternative, the state of vascularity of the femoral head in general and of the pressure segment in particular is relevant. It has long been considered that a vascular disorder is responsible for the etiology and pathogenesis of osteoarthritis. Ischaemia has been postulated by several workers (Wollenberg 1909; Bürger and Müller 1921; Goldhaft, Wright and Pemberton 1930; Venable and Stuck 1946). If this be true, it would explain to a large extent the impaired local metabolic state of the bone and the lack of availability of minerals to the newly formed matrix. The angiographic studies of Harrison et al. (1953) in osteoarthritic femoral heads, on the contrary, reveal a state of vascular profusion and dilation of blood vessels in the femoral head together with the appearance of new blood vessels. This finding is not consistent with the assumption of a state of locally decreased mineral concentration due to less blood reaching the bone.

The other possibility is of an exceptionally rapid osteoid production with which the local blood stream cannot keep pace in supplying minerals. The large number of osteoblasts around the trabeculae support this hypothesis but we cannot explain the hypertrophy and rapid formation of osteoid. It is known that pre-osteoblasts, the forerunner of osteoblasts, proliferate at sites of imminent osteogenesis and that these are derived from primitive mesenchymal cells which also give rise to fibroblasts, chondroblasts and reticuloblasts. This 
connective tissue metaplasia is presumably at work in the pressure segment of the osteoarthritic femoral head and the hyperactivity can be presumed to have been triggered off by a local histochemical stimulus of unknown nature.

It is not possible to say on the basis of this study what factor or factors may be responsible. Cellular metaplasia, local histochemical mineralisation inhibitor or cell stimulating enzymes, altered local blood oxygen tensions affecting mineral uptake or enhancing cell activity are all matters of conjecture. Tissue cultures, histochemical techniques and labelled mineral studies may help to clear up the mystery.

\section{SUMMARY}

1. Specimens are described in which osteoid was seen in undecalcified bone sections prepared from a number of osteoarthritic femoral heads.

2. It was localised mostly in the pressure segments.

3. The reason for the presence of this osteoid is not well understood and the possibilities of local histochemical changes and/or cellular metaplasia are discussed.

\section{REFERENCES}

BaLl, J. (1957): A Simple Method of Defining Osteoid in Undecalcified Sections. Journal of Clinical Pathology, $10,281$.

Bürger, M., and Müller, E. (1921): Beiträge zur Pathogenese der Knorpelerkrankungen. 1. Úber Nekrose, Verkalkung und Verknöcherung des Ohrknorpels nach Erfrierung. Zeitschrift für die gesamte Experimentelle Medizin, 25, 345.

Collins, D. H. (1966): Pathology of Bone. London: Butterworth \& Co.

Garner, A., and Ball, J. (1966): Quantitative Observations on Mineralised and Unmineralised Bone in Chronic Renal Azotaemia and Intestinal Malabsorption Syndrome. Journal of Pathology and Bacteriology, 91, 545.

Goldhaft, A. D., Wright, L. M., and Pemberton, R. (1930): The Production of Hypertrophic Arthritis by Interference with the Blood Supply. American Journal of Medical Sciences, 180, 386.

Harrison, M. H. M., Schajowicz, F., and Trueta, J. (1953): Osteoarthritis of the Hip: A Study of the Nature and Evolution of the Disease. Journal of Bone and Joint Surgery, 35-B, 598.

Mclean, F. C., and Bloom, W. (1940): Calcification and Ossification. Calcification in Normal Growing Bone. Anatomical Record, 78, 333.

Venable, C. S., and Stuck, W. G. (1946): Muscle-Flap Transplant for the Relief of Painful Monarticular Arthritis (Aseptic Necrosis) of the Hip. Annals of Surgery, 123, 641.

Wollenberg, G. A. (1909): Die Äetiologie der Arthritis deformans im Lichte des Experimentes. Archiv für Orthopädie, Mechanotherapie und Unfallchirurgie, Wiesb., 7, 226.

VOL. 51 B, NO. 2, MAY 1969 